AUTHORS

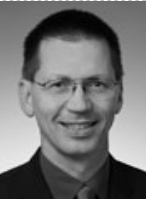

DR.-ING. TILMAN BÜNTE is a Research Associate in the Department of Vehicle System Dynamics at DLR in Oberpfaffenhofen (Germany).

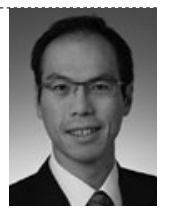

LOK MAN HO, BE/BSC is a Research Associate in the Department of Vehicle System Dynamics at DLR in Oberpfaffenhofen (Germany).

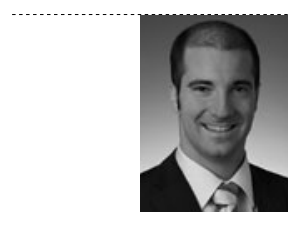

DIPL.-ING. CLEMENS SATZGER is a Research Associate in the Department of Vehicle System Dynamics at DLR in Oberpfaffenhofen (Germany)

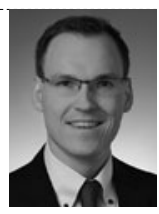

DIPL.-ING. JONATHAN BREMBECK is Head of the Automotive Section of the Department of Vehicle System Dynamics in the Institute of System Dynamics and Control which is part of the Robotics and Mecha-

tronics Center in the German Aerospace Center (DLR Oberpfaffenhofen) in Wessling near Munich (Germany).

\title{
CENTRAL VEHICLE DYNAMICS CONTROL OF THE ROBOTIC RESEARCH PLATFORM ROBOMOBIL
}

The RoboMobil is DLR's space-robotics driven by-wire electro-mobile research platform for mechatronic actuators, vehicle dynamics control, human machine interfaces, and autonomous driving (DLR = German Aerospace Center). Due to its four highly integrated identical Wheel Robots it exhibits an extraordinary manoeuvrability even allowing for driving sideward or rotating on the spot. Topics related to vehicle dynamics control are addressed in this article.

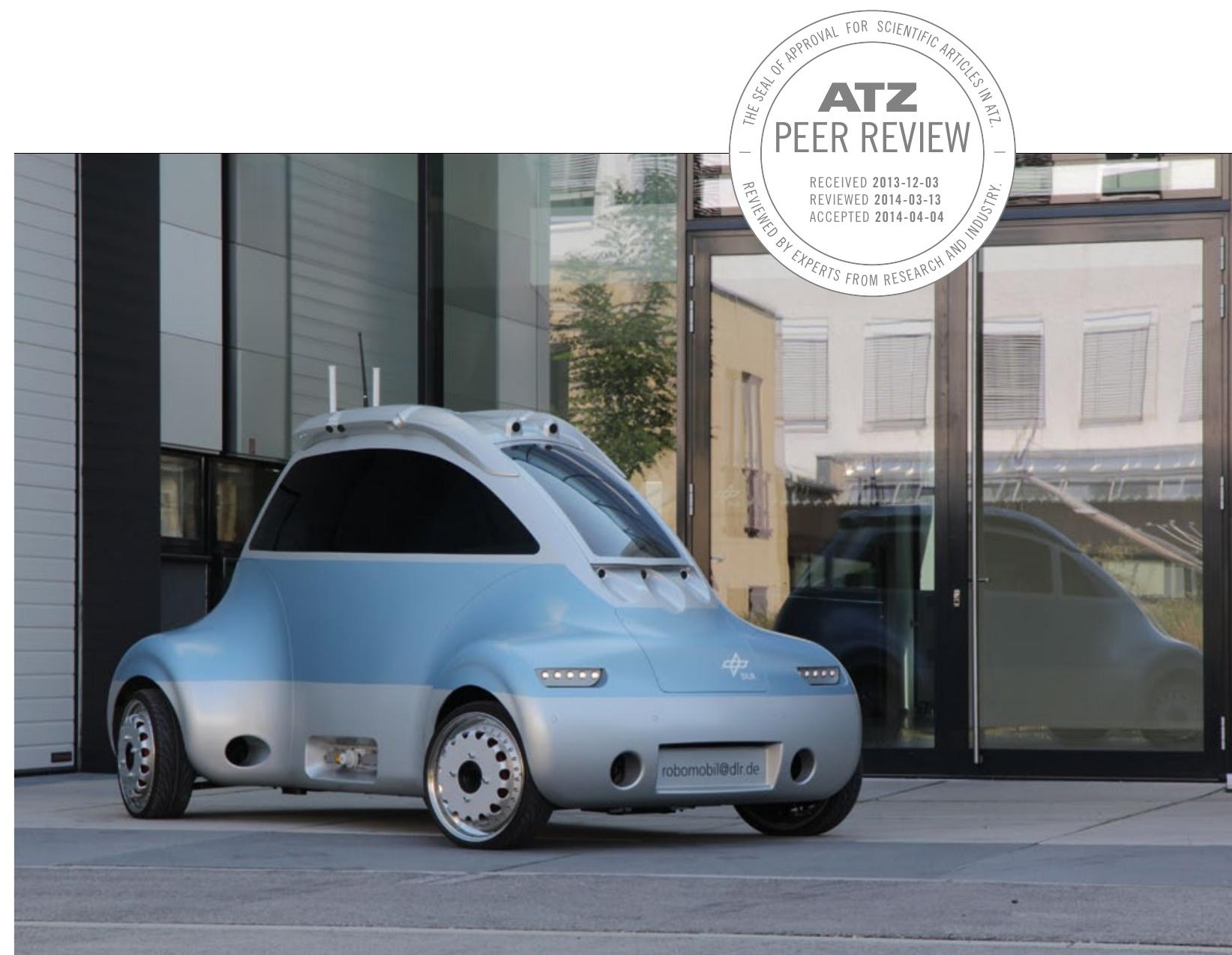


1 THE ROBOMOBIL ALIAS ROMO

2 CHALLENGES AND CONTRIBUTION

3 TOOLS AND METHODS

4 VEHICLE DYNAMICS CONTROL AND INTEGRATED CHASSIS CONTROL 5 OUTLOOK

\section{THE ROBOMOBIL ALIAS ROMO}

On the Romo, central intelligent control on rapid control prototyping $(\mathrm{RCP})$ systems is combined with a rich instrumentation by environmental sensors such as $360^{\circ}$ stereo vision including advanced image processing, inertial measurement unit (IMU) and further vehicle dynamics sensors plus innovative operating controls such as a three degree-of-freedom force-feedback sidestick. Technical details can be found at [1]. The Romo's configuration supports multiple Vehicle Level Applications (VLA) including interactive driving, remote control, and semi or full autonomous driving $[2,3]$. It provides an excellent tool for multi-faceted research in various scientific areas such as integrated vehicle dynamics control (VDC) and automatic driving, human machine interfaces (HMI), advanced driver assistance systems, Car2X communication, energy management, and fault-tolerant control. Developed under the lead of the Robotics and Mechatronics Center (RMC) at the German Aerospace Center (DLR), the Romo's clean sheet, purpose design approach and the abandoning of road homologation enable the exploration of advanced robotics technologies. The concepts and paradigms for robotic systems, which are of the scientific expertise of DLR, can be demonstrated in terms of their applicability to road vehicles. Since its maiden voyage in September 2010, the mechatronic two-seater has been performing experiments on test rigs and open-air proving grounds, and was awarded the "visionary product concept" at eCarTec 2012.

The RoboMobil is propelled by permanent magnet synchronous machines integrated as in-wheel motors (IWM) in each of the Wheel Robots, (1. Each of these motors provides up to $160 \mathrm{Nm}$ and allows tractive and recuperative power of $16 \mathrm{~kW}$ per wheel. A lithium-ion battery supplies the direct drive IWMs and stores a maximum of $13 \mathrm{kWh}$ at $350 \mathrm{~V}$. The in-wheel steering actuators allow steering rates of up to $65 \%$. The wheel can turn up to $95^{\circ}$ in one direction. Electro-hydraulically driven disc brakes (EHB) with one actuator per axle provide a maximum wheel braking torque of $445 \mathrm{Nm}$ per wheel. The 18 cameras, which can be combined into different stereo pairs, complement the IMU with integrated dual DGPS and the Correvit system to determine the vehicle states. The driver can independently control the vehicle's three horizontal motion degrees of freedom (DOF) using a force-feedback stick [4]. The stick allows translational deflections in both $\mathrm{X}$ - and $\mathrm{Y}$-directions and rotation about its Z-axis to facilitate ergonomic one-handed control of the vehicle's motion.

The Romo research platform and related research topics are funded in the project Intelligent Mobility by the DLR Space Programme. The vehicle dynamics control, which is developed within the DLR Transport Programme, is implemented and validated on the Romo. The association with the two research programmes supports exploiting the transfer of knowledge from technologies associated with the Mars rover [5] as well as mobile robots and facilitates the research on their application to terrestrial mobility Within the Transport Programme, Romo is one of multiple test carriers in the research topic "Next Generation Car (NGC)". NGC forms the parenthesis about the wide-ranging road vehicle research cooperation among a number of DLR research institutes at various sites.

\section{CHALLENGES AND CONTRIBUTION}

The Romo offers extraordinary features from the vehicle dynamics point of view: Using X-by-wire technology, all four steering angles and drive/brake torques can be controlled individually by their respective actuators. Advanced vehicle dynamics control techniques are needed to exploit the full potential of the highly over-actuated vehicle. The steering angles of the both front/rear and left/right axisymmetrically aligned wheel robots can reach $25^{\circ}$ in one direction and $95^{\circ}$ in the other, giving the Romo a superior manoeuvrability. Neglecting tyre side slip for this basic geometric consideration, the instantaneous centre of rotation of the horizontal motion $\mathrm{ICR}_{\text {geo }}$ can only reside in the regions which are depicted medium grey in 2. They are determined as follows. The steering angle limits of each individual wheel result in infeasible regions for the $I \mathrm{CR}_{\text {geo }}$ (which must lie in the extension of the wheel axis). Those of the front left wheel are exemplarily depicted in light red. The valid (i.e. medium grey) $I C R_{\text {geo }}$ regions for the whole vehicle are obtained after forming the complement of the set union of all invalid regions from all wheels. From the multiple $\mathrm{ICR}_{\text {geo }}$ regions one can derive three operational motion control modes [6]:

1. ongitudinal (i.e. normal) driving

2. lateral motion

3. rotation.

Each of the motion control modes plus transition between the modes at vehicle standstill demand its own specific HMI concept.

While X-by-Wire and mechatronic systems present opportunities to improve vehicle dynamics control, the random failure modes of the $E / E$ subsystem and the deterministic nature of software have to be dealt with. This is of paramount relevance to ensure system safety. Addressing these challenges require advances in fault management and fault tolerant control, both research topics in the RoboMobil project [7].

\section{TOOLS AND METHODS}

Virtual simulation tools are used for the development of the vehicle itself as well as the vehicle dynamics control. The virtual design and testing environment comprises detailed multi-body vehicle models based on the object oriented modeling language Modelica.

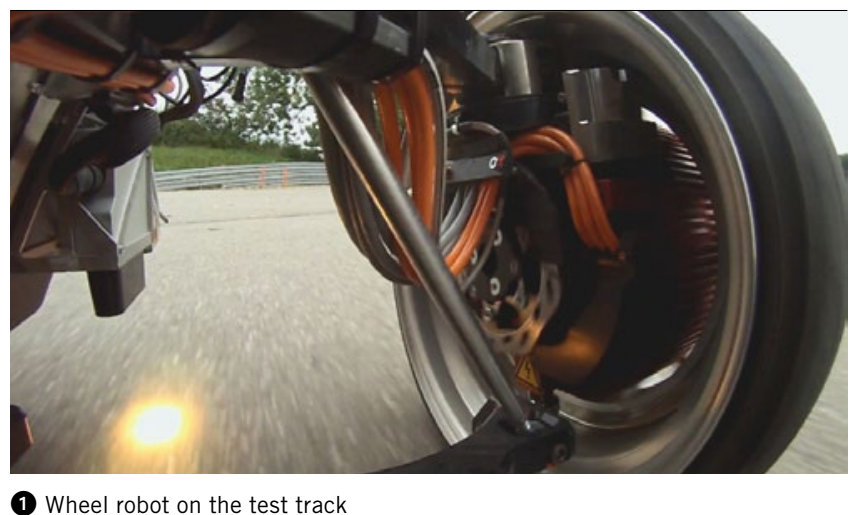




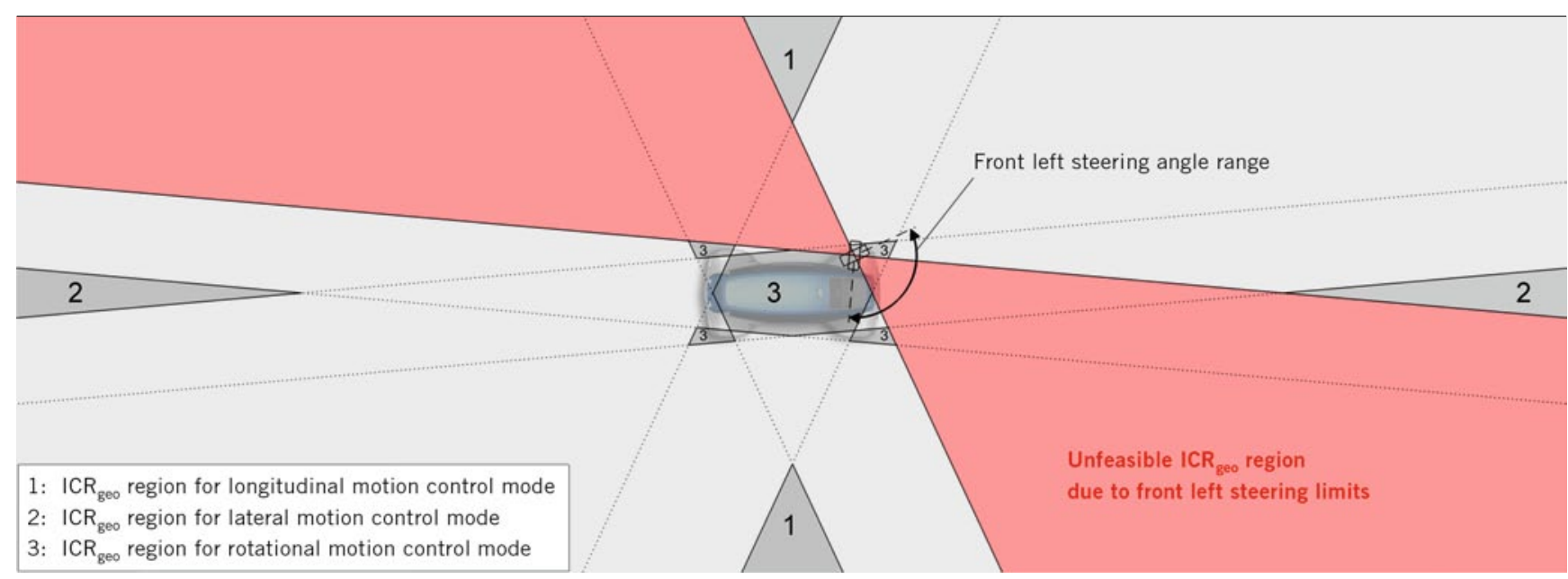

2 Motion control modes according to the regions for possible geometric ICR locations [6]

They include component models for sensors, electro-mechanic actuators plus procedures for automated execution and post-processing of standard and non-standard driving tests. During vehicle dynamics control development the real-time capable plant models are co-simulated with Simulink control software using the functional mock-up interface (FMI) standard. For control design DLR makes extensive use of models from the comprehensive Modelica libraries of DLR while exploiting proven techniques from aerospace and robotics such as dynamic inversion and offline optimization of controller parameters. For the validation of the software and control algorithms on the target controller hardware, a dSpace Scalexio based HiL platform simulates the communication interfaces and vehicle dynamics including actuators and sensors. The software can be tested in a wide range of virtual driving situations and manoeuvres before it is applied on the real vehicle.

Real time optimisation is a technique which is used in different places in the control software, e.g. for model predictive wheel slip control or control allocation. Recent advances enable fast convergence and from that high update rates of the global chassis control. The hierarchical RCP architecture, (3, reflects the centralised architecture derived from robotics, where decision making, planning, and control of tasks and motions are centrally computed and communicated to smart actuators.

\section{VEHICLE DYNAMICS CONTROL AND INTEGRATED CHASSIS CONTROL}

Feedback control of accelerations, yaw rate and chassis side slip angle is applied to the Romo only in its longitudinal motion control mode. In the other modes (which are not detailed here) due to low speeds the effects of tire slip and external disturbances are negligible. The scalable control concept of DLR complies with the standard notion of mobile robotics which presumes the task of following a given path, 4. Accordingly, a common "kinematic motion demand" interface for describing the horizontal motion, 5, is defined. Independent of the VLA it is a vector composed of three scalars: One of them is dedicated to the pure longitudinal dynamics of the vehicle along the given path, given by either (tangential) speed or acceleration. The other two elements refer to the geometry of the motion, namely the curvature $\rho$ and the chassis side slip angle $\beta$. For good feed-forward control performance, these signals should be smooth and be supplied with a sufficient number of partial derivatives w.r.t. the arc length s.

Corresponding to the X-by-wire nature of the Romo and the robotics inspired control architecture, its vehicle dynamics control is organised in a hierarchic and centralised way as depicted in (4). The actuator control is executed at high sample rate on their respective control units. The upper three layers in the pyramid are executed on the Central Controller from (3). The VLA includes path planning and path following in the case of autonomous driving or reading and serving the $\mathrm{HMI}$ in case of interactive driving. The latter can be conducted by the operator either sitting in the car or by remote control (teleoperation). The VLA that supports autonomous or shared autonomous driving is named Artificial Intelligence Agent (AIA) [8]. In shared autonomy, the driver provides only a rough motion demand which is refined with anticipation and executed by the AIA using advanced path planning and path following algorithms. Opposite to conventional driver assistance systems, the binding motion demand is given by the AIA. A unified interface enables seamless transition between manual and autonomous driving. Every VLA provides the previously explained kinematic motion demand to the second layer. Each of the three lower hierarchy levels reports the current actuation, force, or motion capabilities to the higher level in order to facilitate optimal exploitation of the limits there. DLR realises each level in various degrees of refinement which can be interchanged, making the whole concept scalable.

An essential module which allows a basic feed-forward actuator control is called the Geometrically-based Vehicle Dynamics Control (GeoVDC). It also acts as the feed-forward part of the more sophisticated implementations. The curvature $\rho$ and the chassis side slip angle $\beta$ can both be controlled independently along the path's arc length s due to the possibility of rear wheel steering on the Romo. The instantaneous centre of rotation ICR, expressed in the vehicle coordinate system, can be calculated, (4) (left) directly from the motion demand Eq. 1:

EQ. $1 \quad\left[\begin{array}{l}x_{/ K R} \\ y_{I C R}\end{array}\right]=\frac{1}{\rho-\partial \beta / \partial s}\left[\begin{array}{c}-\sin (\beta) \\ \cos (\beta)\end{array}\right]$




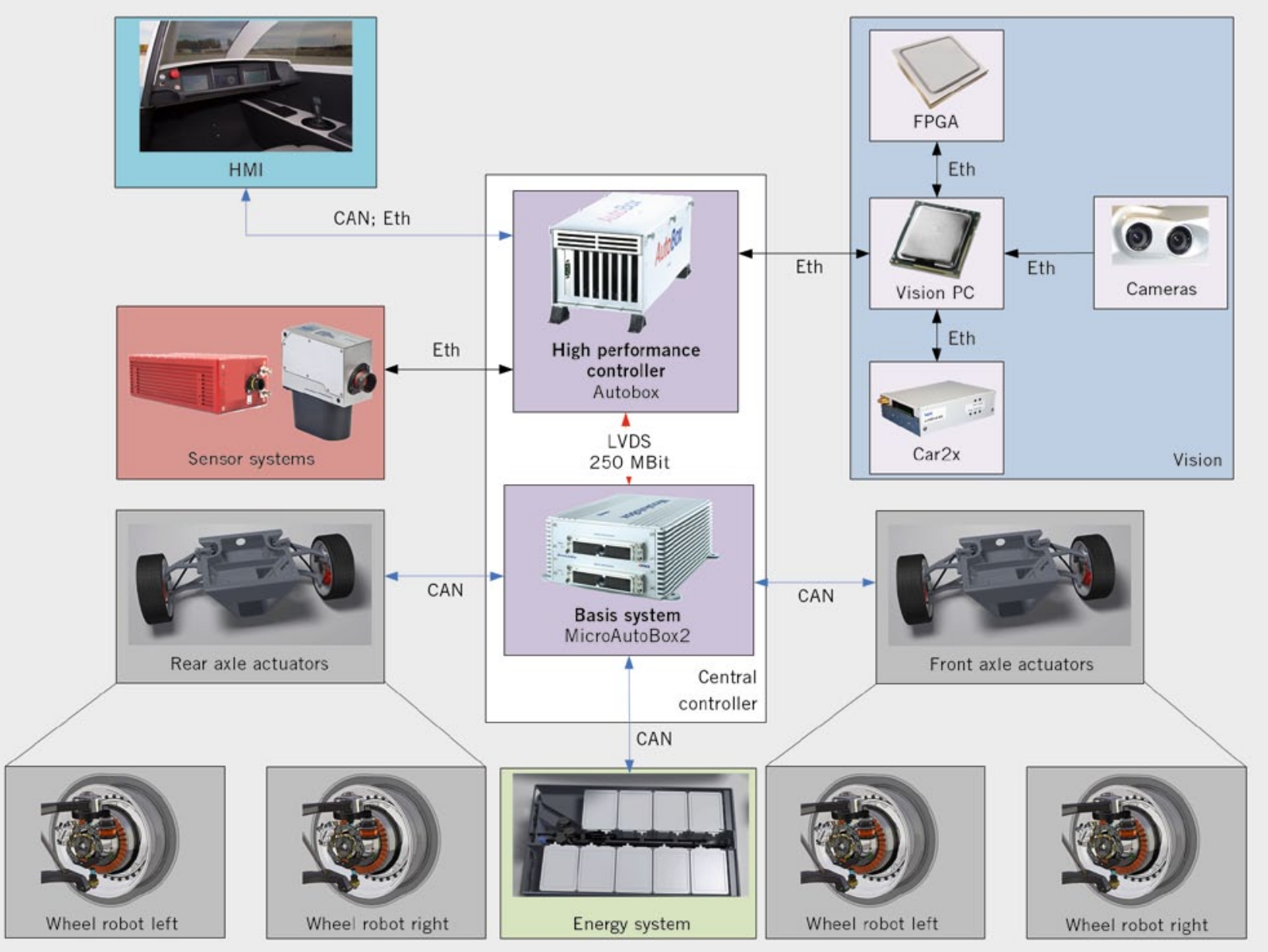

3 Romo IT network architecture

This link provides the basis for a feed-forward control of the steering angles. They are set such that the extended wheel axes pass through $\mathrm{ICR}_{\text {geo }}$ which forms a geometric approximation of the ICR neglecting tire side slip. In a preceding step $\mathrm{ICR}_{\text {geo }}$ is calculated from the ICR corresponding to the motion demand in (1). For this purpose a speed-scheduled steady state single track model accounting for tire slip, (4) (right) is applied. The speed-dependent boundaries of the allowed ICR-region are continuously computed
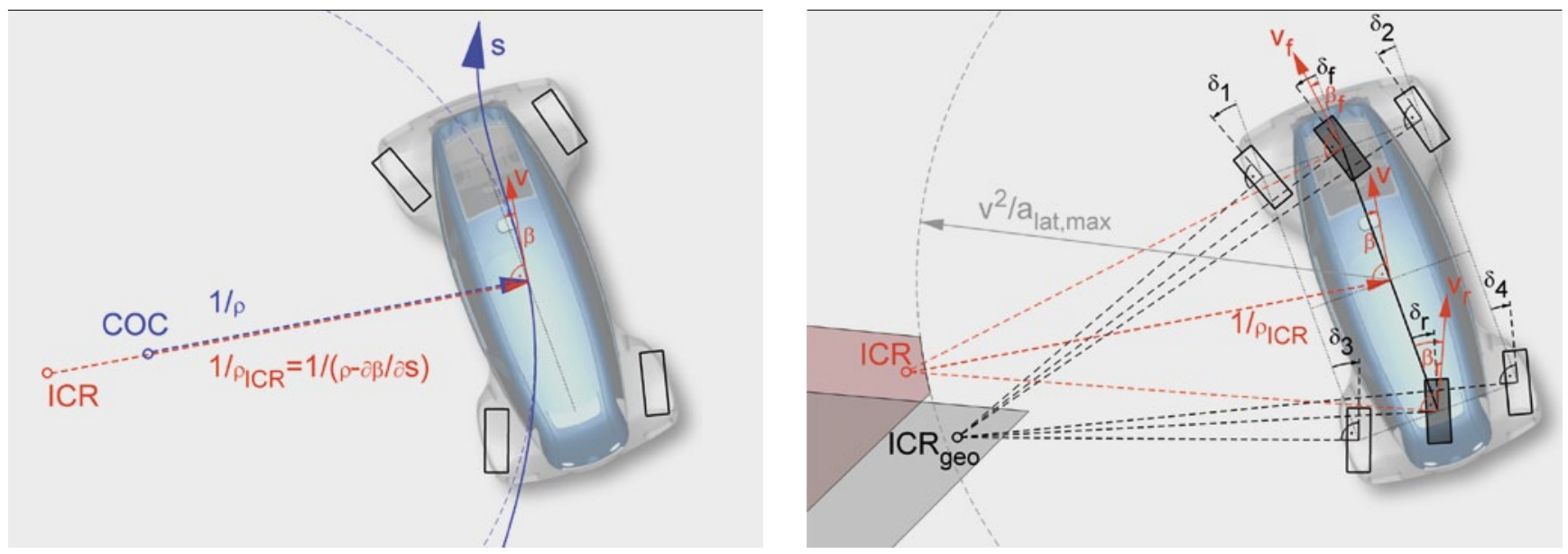

Longitudinal motion control mode; path based motion demand (left), feed-forward control accounting for tyre side slip as of the linear single track model and using geometric steering angle distribution (right) 


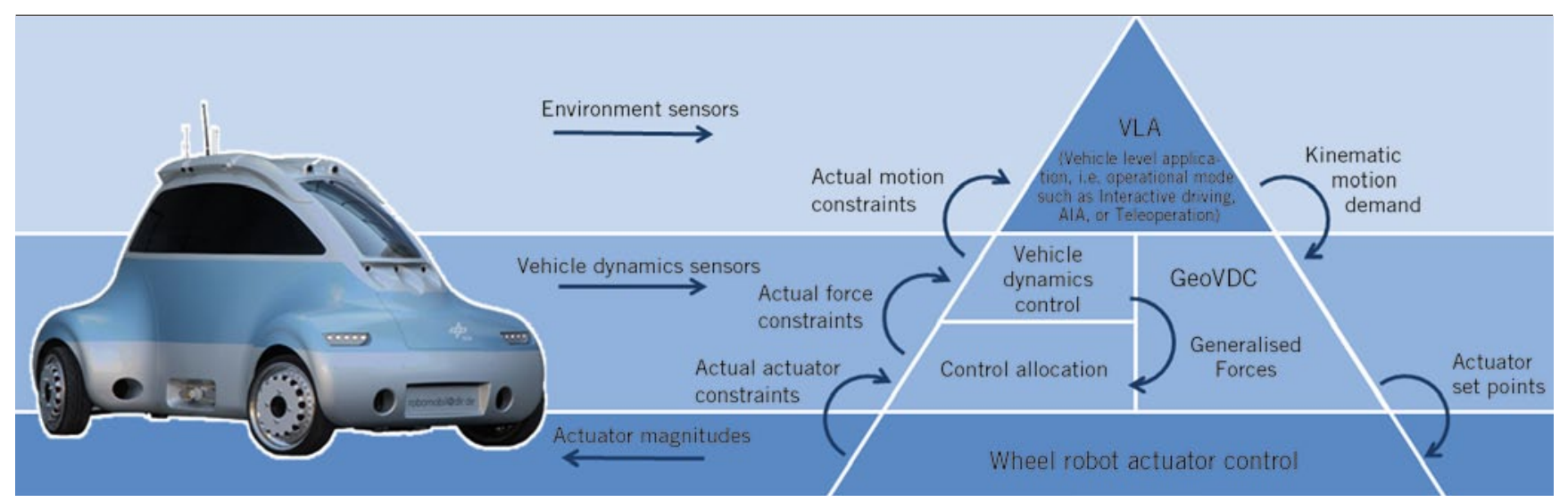

5 Scalable vehicle dynamics control architecture for longitudinal motion control mode

for use by the HMI to calculate scaling and/or force-feedback and for the AIA path planning. Together with a feed-forward computation of the wheel drive or brake torques (similar to the steering angles) the GeoVDC is a simple implementation of the joint two middle levels in the scalable control concept. Using GeoVDC and moderate driving manoeuvres and nominal vehicle parameters assumed, DLR already achieves respectable accuracy of the actual motion compared to the kinematic motion demanded. However, due to the limited validity of the linear single track model, operation while approaching the stability limits of the vehicle requires additional and more sophisticated control action. Classical feedback of vehicle dynamics measurements ("vehicle dynamics control" layer in (4)) such as yaw rate or lateral acceleration and optionally chassis side slip angle is used to improve control performance and driving safety in the presence of model imperfection or external disturbances.

Regarding the three horizontal motion degrees of freedom, the Romo is seven times over-actuated with its ten vehicle dynamics actuators. The task of the next layer, control allocation, is to make

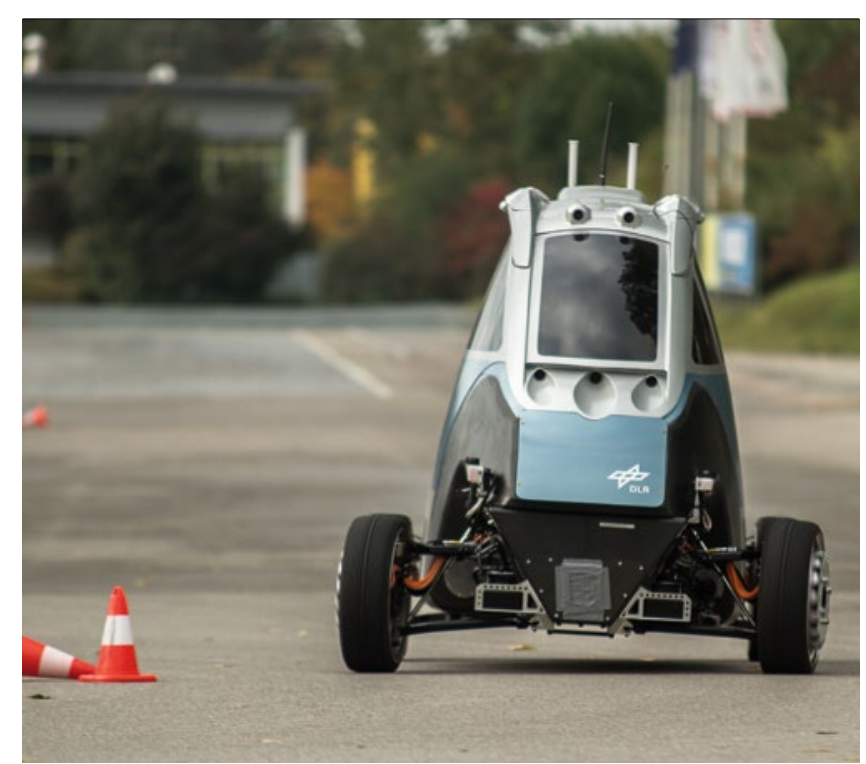

6 Romo in action on ADAC Augsburg proving ground best benefit in real time of the over-actuation such that a motion demand is precisely executed and simultaneously other criteria are optimized such as energy efficiency, safety, and comfort. Henceforth DLR refers to integrated chassis control rather than to vehicle dynamics control. The research combines inverse vehicle dynamics models with real time optimisation [5] at which the simultaneous consideration of various criteria is achieved by including terms in the cost function accounting for each of them. For energy efficiency, an additional term is included in the cost function accounting for actuator losses and power consumption for driving along the planned path [10].

To deal with actuator failure the reduced capabilities of a faulty actuator are communicated from the fault diagnostic module and the cost function is adapted to reflect degraded performance targets. In this way and according to the overall diagnosis, the fault tolerant vehicle dynamics controller is recon\#d [9]. In the future, vertical dynamics control will also be applied by means of high bandwidth FPGA controlled semi-active dampers. Subordinate to the previously described control allocation, longitudinal tire slip control aims to prevent wheel blocking and skidding. The IWMs act as fast and energy efficient complements to the EHBs [10]. The hybrid brake system formed by these actuators is exploited for optimal trade-off between energy recuperation and braking effect, using a brake blending algorithm based upon model predictive control allocation (MPCA).

Finally, vehicle state estimation is an important issue to facilitate integrated chassis control. A concept for a generic Modelica based observer featuring automatic code generation was developed and implemented. It presumes a continuous (usually nonlinear) Modelica model of the plant. The approach utilizes the Functional Mockup Interface (FMI) standard for model exchange und co-simulation and enables the application of different observer designs, like EKF and UKF nonlinear Kalman Filters [11].

6 shows the Romo with dismounted fairings in a dynamic driving manoeuvre using GeoVDC. Yaw rate and chassis side slip angle signals, each with corresponding steering angles are displayed in $\boldsymbol{(}$ and $\boldsymbol{8}$, respectively. During the presented experiments, the particular set points were manually commanded with the $\mathrm{HMI}$ stick. From these results, one can verify a good agreement between the motion demands and the measurements, which demonstrates that the GeoVDC is a sufficient mean to control the Romo's lateral dynamics for moderate motion demands. Similarly, 

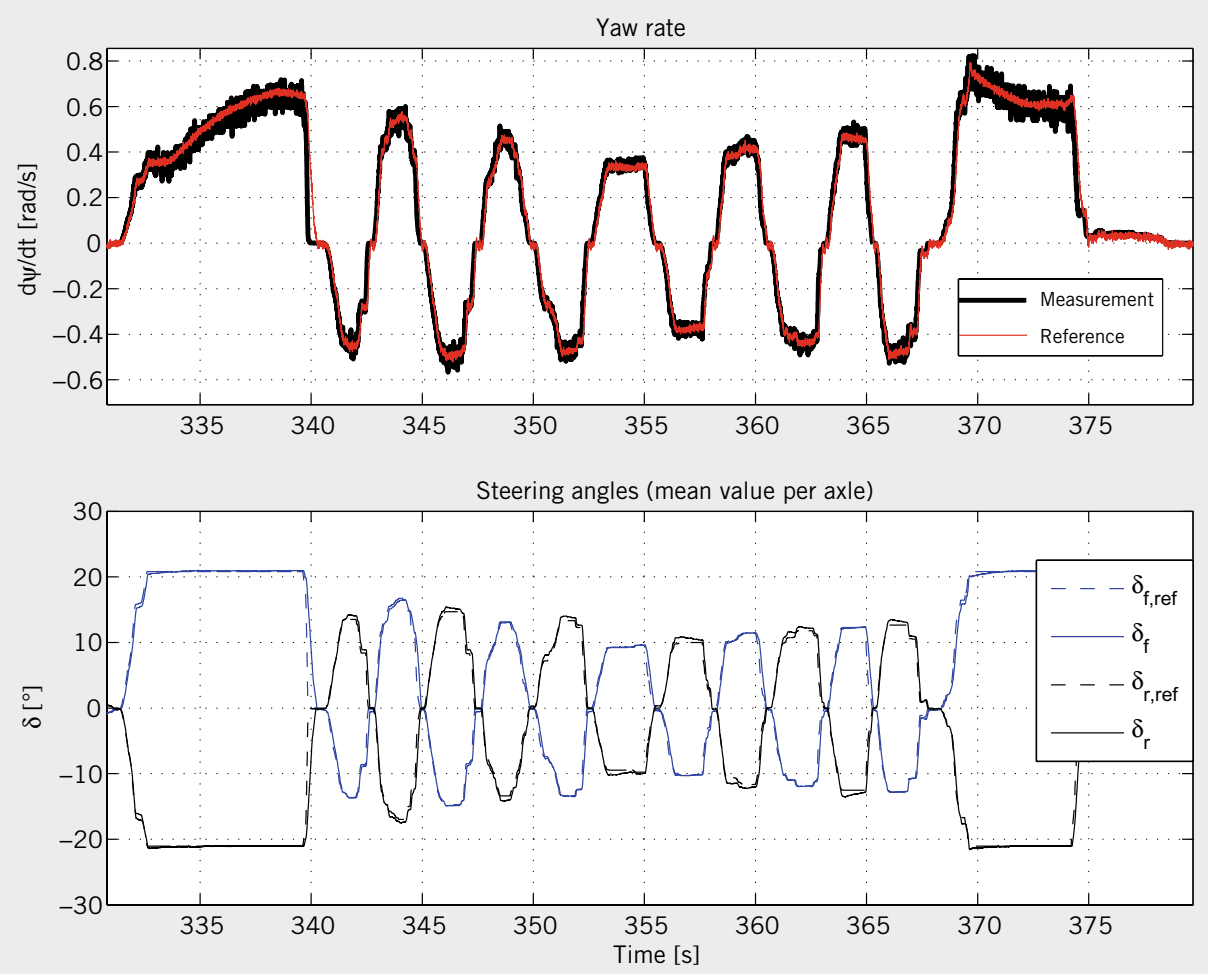

7 Experimental validation: yaw rate and steering angles
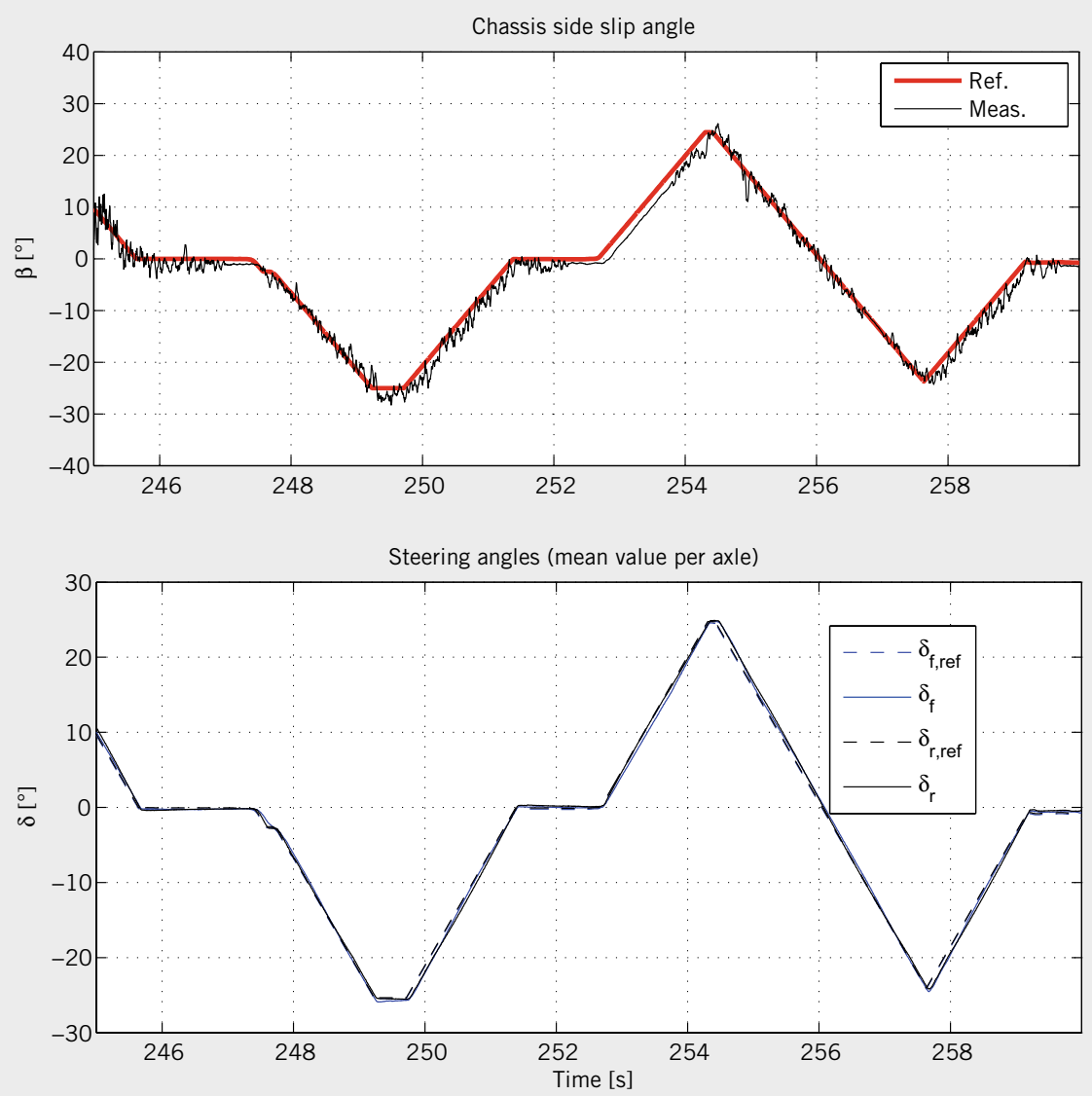

8 Experimental validation: chassis side slip angle and steering angles 


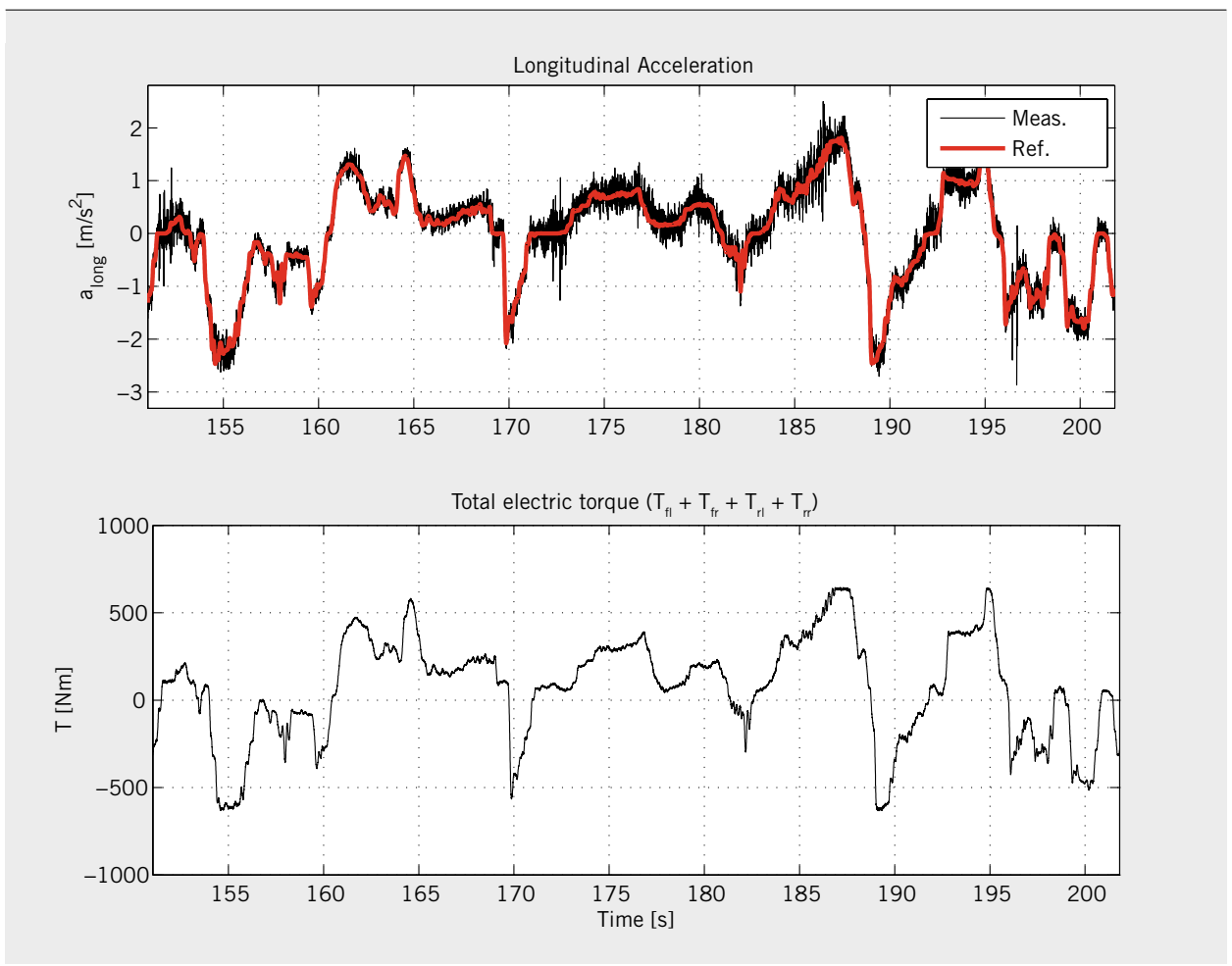

9 Experimental validation: ongitudinal acceleration the control of the Romo's longitudinal motion, depicted in $\mathbf{9}$, also reveals a good tracking performance of the acceleration demands.

\section{OUTLOOK}

There is a wide range of research planned with the Romo. One of the long-term visions of DLR is the usage as an "in-drive-simulator" to emulate novel vehicles' dynamics in their early phase of virtual development. For this purpose, the control framework will be generalized to make it flexibly adaptable to various kinds of vehicle actuation configurations. In the near future DLR plans to conduct further experiments for parameter identification, integrated chassis control validation, autonomous driving, and path following control. Integration of up-to-date environment information (e.g. as acquired by Car-to- $X$ ) as well as sensorless tire contact force estimation are intended to support the time critical chassis control algorithms.

\section{REFERENCES}

[1] DLR Robotics and Mechatronics Center: The Robotic Electric Vehicle. URL http://www.dlr.de/rm/robomobil

[2] Schaub, A.; Brembeck, J.; Burschka, D.; Hirzinger, G.: Robotisches Elektromobil mit kamerabasiertem Autonomieansatz. In: ATZelektronik 6 (2011), No. 2 , pp. 10-17. URL http://dx.doi.org/10.1365/s35658-011-0023-8

[3] Schaub, A.; Brembeck, J.; HO, L. M.; Sattzger, C. ; Tobolar, J.; Bals, J.; Hirzinger: ROMO - The Robotic Electric Vehicle. In: 22 ${ }^{\text {nd }}$ IAVSD International Symposium on Dynamics of Vehicle on Roads and Tracks, 2011

[4] Weber, B. ; Panzirsch, M.; Preusche, C.; Deml, B.: Der aktive Sidestick als Bedienelement im Elektromobil. In: Schütte, M. (Hrsg.): Mensch, Technik, Organisation - Vernetzung im Produktentstehungs- und -herstellungsprozess. Vom 23.-25. March 2011. Als Ms. gedr. Dortmund: GfA-Press, 2011 (Jahresdokumentation/Gesellschaft für Arbeitswissenschaft e.V, 2011)

[5] Krenn, R.; Renn, R; Köppern, J.; Bünte, T.; Brembeck, J.; Gibbesch, A.; Bals, J.: Modellbasierte Regelungsansätze für überaktuierte planetare Rover und robotische Elektromobile. In: at - Automatisierungstechnik 61 (2013), No. 3, pp. 183-194
[6] Bünte, T.; Brembeck, J.; Ho, L.M.: Human machine interface concept for interactive motion control of a highly maneuverable robotic vehicle. In: 2011 IEEE Intelligent Vehicles Symposium (IV), pp. 1170-1175

[7] Ho, L.M.: Application of Adaptive Thresholds in Robust Fault Detection of an Electromechanical Single-Wheel Steering Actuator. In: $8^{\text {th }}$ IFAC Symposium on Fault Detection, Supervision and Safety of Technical Processes (SAFEPROCESS), 2012

[8] Schaub, A.; Hellerer, M.; Bodenmüller, T.: Simulation of Artificial Intelligence Agents using Modelica and the DLR Visualization Library. In: $9^{\text {th }}$ International Modelica Conference, Munich, Germany: Linköping University Electronic Press, 2012 (Linköping Electronic Conference Proceedings), pp. 339-346

[9] You, S. (Hrsg.); Jalics, L. (Hrsg.): Hierarchical Component-based Fault Diagnostics for By-Wire Systems, 2004

[10] Satziger, C.; Brembeck, J.; Otter, M.: Framework for the Evaluation of Wheel Torque Blending Algorithms. In: Advances in Automotive Control: Elsevier, IFAC, 2013 (IFAC proceedings volumes), pp. 347-352

[11] Brembeck, J.; Zimmer, D.; Otter, M.: Nonlinear Observers based on the Functional Mockup Interface with Applications to Electric Vehicles. In: $8^{\text {th }}$ International Modelica Conference, 2011 